patients in the UK. We examined the records of 11120 patients and assembled a longitudinal, retrospective cohort from those seen between 2007 and 2015. We used Cox proportional hazard models to assess the survival of patients on NIV.

Results 1077 patients (715 adults and 362 children $<16$ years) had reported use of NIV recorded at least once. Usage increased after 2012 (figure 1). At the first recorded use of NIV the median (IQR) age was 21 years $(14,28)$, BMI 18.4 $\mathrm{kg} / \mathrm{m}^{2}(16.8,22.7), 49.2 \%$ were male, $90.3 \%$ on PERT, $75.1 \%$ growing Pseudomonas, 54.6\% homozygous F508del; the mean FVCpp was $64.5 \%$ and $\mathrm{FEV}_{1}$ pp $47.2 \%$. At this time $68.8 \%$ of patients had a $\mathrm{FEV}_{1}$ pp $<60 \%$; 52\% were $<40 \%$, while in adults this percentage reached $61 \%$. In children there was a higher proportion starting treatment with better lung function ie $\geq 60 \%$ (33.8\%). The median survival of patients who start NIV is 3.47 years. The hazard ratio for NIV use was 3.90 (95\% CI: 3.06-4.96).

Conclusions Not surprisingly, patients start NIV when their lung function is significantly impaired. Yet, increased proportions of people with $\mathrm{FEV}_{1} \mathrm{pp} \geq 40 \%$ on NIV were also identified. The higher lung function at the start of NIV for children may reflect that it is used for purposes other than a bridge to transplant in this group; the registry only collects a yes/no variable for NIV use and not the reason for use. Survival after initiation of NIV is poor; this is likely reflect that NIV is a marker of disease severity but further analysis will be needed to explore this.

\section{P251 ENVIRONMENTAL FUNGAL SAMPLING IN A CYSTIC FIBROSIS CENTRE}

${ }^{1} \mathrm{~L}$ Collier, ${ }^{1} \mathrm{RJ}$ Bright-Thomas, ${ }^{2} \mathrm{M}$ Richardson, ${ }^{1} \mathrm{AM}$ Jones. ${ }^{1}$ Manchester Adult Cystic Fibrosis Centre, Manchester, UK; ${ }^{2}$ Mycology Reference Centre Manchester, Manchester, UK

\subsection{6/thoraxjnl-2017-210983.393}

Objectives Aspergillus is a ubiquitous organism and CF lungs are vulnerable to infection. Aspergillus is known to be found in high numbers in organic matter and during building works. We commenced environmental fungal air sampling prior to and during building work carried out adjacent to our CF unit to evaluate and monitor our air quality.

Methods An SAS Microbial air sampler sampled 1 cubic metre of air over $5 \mathrm{~min}$ in assigned locations throughout our ward on a weekly basis. Outdoor samples were taken during this time for comparison. Each plate was cultured for 4 days at $30^{\circ} \mathrm{C}$.

Results The predominant organism was Aspergillus fumigatus, the second Penicillium Spp. Site 1, outdoor air: revealed a maximum yield of 59 colony forming units (CFU) A. fumigatus (range 0-59 CFU, median 9), and 8 CFU Penicillium (08, median 0). Site 2, ward corridor: 29 CFU A. fumigatus (029 CFU, median 2.5), 15 CFU Penicillium (0-15, median $0.5)$. Site 3, patient room: $12 \mathrm{CFU}$ A. fumigatus (0-12 CFU, median 2), 9 CFU Penicillium (0-9, median 0). Site 4, positive pressure anteroom: $2 \mathrm{CFU}$ A. fumigatus (0-2 CFU, median 0), 1 CFU Penicillium (0-1 CFU, median 0). Site 5, patient room: $58 \mathrm{CFU}$ A. fumigatus (0-58 CFU, median 4.5), $5 \mathrm{CFU}$ Penicillium (0-5 CFU, median 2.5). There was a clear rise in
Aspergillus yield demonstrated during the summer months in all areas except our positive pressure anteroom which persistently yielded negligible fungal growth.

Conclusions High levels of A. fumigatus were persistently yielded from sites 1, 2, 3 and 5, both at baseline and during building works, with peak counts being found in the summer months. Fungal ingress onto the ward was demonstrated in all sites except in our positive pressure anteroom, with $\geq 10$ air changes per hour, leading to isolation rooms. This gives doubts about the efficacy of our ventilation system in the majority of locations throughout our ward and needs to be clinically correlated with patient outcomes.

\section{P252 CLINICAL PROFILE OF INFANTS WITH CYSTIC FIBROSIS SCREEN POSITIVE, INCONCLUSIVE DIAGNOSIS (CFSPID) IN THE WEST MIDLANDS}

SH Ali, J Clarke, S Rao, M Desai, P Nagakumar. Birmingham Women's and Children's Hospital, Birmingham, UK

\subsection{6/thoraxjnl-2017-210983.394}

Background The introduction of newborn screening for cystic fibrosis (CF) has resulted in up to $10 \%$ of infants with the positive result but inconclusive diagnosis. The European cystic fibrosis consensus group has defined CFSPID as newborn screened infants with elevated immunoreactive trypsinogen and normal sweat chloride and two CFTR mutations, at least one with unclear phenotypical consequences OR infants with intermediate sweat chloride with one or no CFTR mutations (Munck:2015). The majority will remain well and have no long-term health implications. However, a small percentage of these infants may develop the disease by three years.

Aim To study the prevalence, clinical characteristics and clinical course of children who meet the CFSPID criteria within a large UK regional paediatric CF centre

Methods Retrospective data review of children identified on newborn screening who met the ECFS CFSPID criteria using $\mathrm{CF}$ registry, regional screening centre notification and the local network database.

Results Between October 2006 to December 2016, 148 infants were CF screen positive. 9 children (6.08\%) met CFSPID diagnostic criteria. The median follow up period was 25 months (range 6-95 months). Median age at repeat sweat test was 3 years (range 1.5-5 year). All patients were heterozygous for $\Delta \mathrm{F} 508$. The second mutation identified included $\mathrm{R} 117 \mathrm{H}$ in 6 children, D1152H in one child and Arg810Ser in 2 siblings. 2 patients had Staph aureus and Haemophilus influenzae once on cough swabs. None of them was positive for Pseudomonas aeruginosa. All were pancreatic sufficient. All children in the group remained nutritionally well. None had chronic isolates of significant respiratory pathogens common in cystic fibrosis and repeat sweat test remained normal in all patients. Conclusion Early outcomes for CFSPID are good. National guidance on information provided to families at diagnosis, frequencies of clinic follow up are necessary. In addition assessment of the psychological impact of the diagnosis to the families would be valuable. National data collection possibly through a UK CF registry based study to inform long term outcomes in children with a diagnosis of CF SPID is needed. 


\section{P253}

INVESTIGATING THE COMPLEXITY OF THE RELATIONSHIP BETWEEN GASTRO-OESOPHAGEAL REFLUX AND CF LUNG DISEASE

${ }^{1} \mathrm{RW}$ Lord, ${ }^{2} \mathrm{~S}$ Treadway, ${ }^{2} \mathrm{JS}$ Pearson, ${ }^{1} \mathrm{PJ}$ Barry, ${ }^{1} \mathrm{~B}$ Bianco, ${ }^{2} \mathrm{PJ}$ Whorwell, ${ }^{2} \mathrm{RB}$ Jones, ${ }^{3} \mathrm{PS}$ McNamara, ${ }^{4} \mathrm{R}$ Beynon, ${ }^{2} \mathrm{JA}$ Smith, ${ }^{1} \mathrm{AM}$ Jones. ${ }^{1}$ Manchester Adult Cystic Fibrosis Centre, Manchester, UK; ${ }^{2}$ University Hospital South Manchester, Manchester, UK; ${ }^{3}$ Alder Hey Childrens Hospital, Manchester, UK; ${ }^{4}$ University of Liverpool, Liverpool, UK

\subsection{6/thoraxjnl-2017-210983.395}

Background There has been a suggested connexion between gastro-oesophageal reflux (GOR) and CF lung disease. Lung disease can result in increasingly negative inspiratory thoracic pressures. These create gastro-oesophageal pressure gradients along which gastric contents may move. Then if aspirated reflux may have the potential to cause lung damage. We aimed to assess if there is a relationship between GOR and markers of lung disease severity.

Methods We are conducting a prospective observational study in stable adult CF patients, measuring GOR with combined $\mathrm{pH}$ and impedance (pH-MII). In preliminary analyses, we have compared reflux measures (total, proximal, supine and supine proximal events) and retrospective data including routinely collected lung function and number of courses of intravenous antibiotics.

Results 51 patients were recruited with 36 patients (mean age 30 years, mean FEV1 54\% predicted, 28 males) completing all measures. Total number of reflux episodes were increased in 58\% (median 81, IQR 55-105) compared to established normative values ( $<75$ episodes). 'High risk' reflux (increased proximal or supine proximal events) was noted in $47 \%$. Increasing reflux and higher number of IV courses in the preceding year displayed a positive trend $(r=0.300, p=0.075)$, but this relationship was not seen for number of courses over 2 years. Curiously, patients with greater numbers of reflux events exhibited less decline in lung function over the preceding year $(r=0.416, p=0.016)$. None of the reflux measures were correlated with baseline lung function.

Conclusions We have demonstrated that patients with stable CF lung disease have high rates of total reflux events in comparison to normative values. Almost half have reflux which has the potential to be high risk for aspiration. There was a suggestion that increased reflux events maybe associated with increased exacerbation risk. However our data also suggests, in complete contrast, that increased reflux relates to less progression of lung disease - this is not in keeping with commonly held beliefs. Further studies will be needed to unpick what is undeniably a complex relationship.

\section{P254 FEASIBILITY OF ULTRASHORT ECHO TIME (UTE) MRI TO EVALUATE THE EFFECT OF LUMACAFTOR/IVACAFTOR THERAPY IN CHILDREN WITH CYSTIC FIBROSIS (CF) HOMOZYGOUS FOR F508DEL}

${ }^{1} S$ Nagle, ${ }^{2} \mathrm{AS}$ Brody, ${ }^{2} \mathrm{~J}$ Woods, ${ }^{1} \mathrm{KM}$ Johnson, ${ }^{3} \mathrm{~L}$ Wang, ${ }^{3} \mathrm{G}$ Marigowda, ${ }^{3} \mathrm{D}$ Waltz, ${ }^{4} \mathrm{~J}$ Goldin, ${ }^{5} \mathrm{~F}$ Ratjen, ${ }^{3} \mathrm{C}$ Hug. ${ }^{1}$ University of Wisconsin, Madison, US; ${ }^{2}$ Cincinnati Children's Hospital Medical Centre, Cincinnati, US; ${ }^{3}$ Vertex Pharmaceuticals Incorporated, Boston, US; ${ }^{4}$ David Geffen School of Medicine, Los Angeles, US; ${ }^{5}$ University of Toronto, Toronto, Canada
Objective To evaluate ultrashort echo time (UTE) magnetic resonance imaging (MRI) as a feasible endpoint in a multicenter trial using a subset of patients aged 6 to 11 years with cystic fibrosis (CF) homozygous for the F508del mutation treated with lumacaftor/ivacaftor (LUM/IVA) combination therapy (NCT02514473).

Methods UTE MRI scans were obtained at study baseline $(n=10)$; a second scan was completed at week 24 in 9 subjects (4 active treatment, 5 placebo) at 3 institutions using MRI hardware from 2 vendors. One of the platforms was 3D radial UTE (Johnson et al. Magn Reson Med. 2013;70:12411250); the other was 3D "stack of stars" UTE (Lederlin et al. J Magn Reson Imaging. 2014;40:839-847). Scans were obtained during $5 \mathrm{~min}$ of tidal breathing without the use of intravenous contrast. MRI scans were scored by 2 independent readers using the Brody score after supervised training on UTE MRI scans. Readers were blinded to time point and treatment group. Scores are presented as mean (SD); no statistical testing was performed.

Results Despite the lower image quality of MRI compared with computed tomography, potential treatment effects were seen on the UTE MRI images (figure 1A). Mean total Brody score decreased from 41.1 to 32.5 with treatment, a change from baseline (SD) of 8.4 (11.2) with active treatment, but increased from 31.3 to 34.6 , a change from baseline of 3.3 (8.2) with placebo (figure 1B). Mucus plugging subscore decreased by 5.0 (5.1) from 8.5 to 3.5 with active treatment but increased by 1.4 (4.4) from 4.2 to 5.6 with placebo. There were no noticeable changes in other subscores (bronchiectasis, peribronchial thickening, parenchyma, or hyperinflation).

Conclusion In this analysis from an exploratory substudy in patients with CF aged 6 to 11 years homozygous for F508del, UTE MRI was a feasible approach for detecting the effect of LUM/IVA, despite the small sample size, short duration of treatment, and limitations in image quality. As optimisation in UTE MRI technology improves image quality, monitoring disease course in patients with CF may improve.

Please refer to page A259 for declarations of interest in relation to abstract P254.

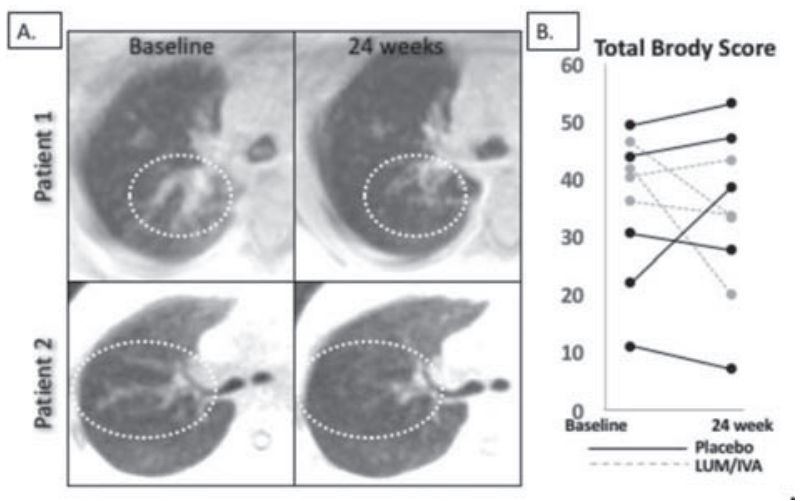

Abstract P254 Figure 1 